\title{
Intelligent communication systems and networks (MLICOM 2017)
}

\author{
Xuemai $\mathrm{Gu}^{1}$
}

Published online: 19 October 2019

(C) Springer Science+Business Media, LLC, part of Springer Nature 2019
Researches show that with the rapid development of wireless communication technology and the large increase in the number of wireless users, current wireless communication technologies cannot meet the communication needs of future wireless users. Therefore, in order to better meet the needs of future wireless users, there is an urgent need for more intelligent wireless communication technologies. Intelligent communication systems and networks make communication networks to evolve from the simple transfer and exchange of information to the intelligent storage and processing of information. The intelligent communication system and network introduce more artificial intelligence in the communication network, thereby improving the service processing capability and adaptability of the communication network, and providing services required for various users at any time. It is a system that can generate and implement new services quickly, easily, flexibly, economically and efficiently. Therefore, intelligent communication systems and networks have great room for development in making wireless communication systems intelligent.

To improve the bit error rate (BER) performance of physical-layer network coding (PNC) in data length asymmetric two-way relay channels (TWRC), a new PNC scheme named combined denoise-and forward and superposition coded physical-layer network coding (DNF-SCPNC) is proposed, and the decoding algorithm of the scheme is improved. Theoretical analysis and simulation results show that DNF-SC-PNC can provide better BER performance and better throughput performance when the data length is asymmetric. In addition, we also demonstrate that the LLR algorithm can provide better performance than the SIC algorithm in data length asymmetric TWRC.

Xuemai Gu

guxuemai@hit.edu.cn

1 Communication Research Center, Harbin Institute of Technology, Nangang District, No. 92 Xidazhi St., Harbin City 150001, Heilongjiang Province, China
Automatic modulation classification (AMC) plays an important role in many fields to identify the type of modulation of a wireless signal in order to recover the signal by demodulation. In this paper, we are dedicated to exploring the appropriate architecture for deep learning methods in the field of communication signal identification. In addition, we also discuss the difficulties of the training algorithm in the deep learning method, and use the transfer learning method to obtain the benefits, thus stabilizing the training process and improving performance.

The nature of multi-hop data transmission in wireless sensor networks will result in severe load imbalances, which, given limited energy resources, will place significant limitations in related applications. Here, a clusterbased balanced energy consumption algorithm (BECA) is proposed, which introduces multiple inter-cluster links to distribute the load, thus achieving global load balancing. The simulation results based on NS2 show that BECA can achieve better balance performance and effectively extend network lifetime.

As one of the most important convergence layer (CL) protocol for delay/disruption-tolerant networking (DTN), Licklider transmission protocol (LTP) is designed for deep space communications, but it has rarely been considered for near earth applications. In this paper, LTP is proposed instead of TCP as CL with Bundle protocol (BP) for future application in GEO-relayed space networks (GRSN). The results show that in transmission efficiency BP with LTPCL outperforms other protocols. Moreover, performance of two different networking schemes, i.e. the onboard DTN routers and the bent-pipe relays are compared. It is observed that bent-pipe relays outperform the on-board DTN routers scheme when the channel condition is good, while the on-board DTN routers scheme performs better with high data loss ratio.

In recent years, several related tracking algorithms have been proposed to exploit the hierarchical features of deep convolutional neural networks. However, most of these methods focus on utilizing layered features for target translation and using fixed size search windows throughout the sequence. In this paper, we propose a new scale 
adaptive tracking algorithm based on hierarchical CNN features, which learns the correlation filter to locate the target and construct a target pyramid around the estimated target position for scale estimation. When the tracking fails, we generate an online detector of the random fern classifier and activate it to re-detect the target.

Micro-Doppler provides valuable information for motion parameter extraction and target recognition of processional warhead targets. In order to solve the estimation problem of these target motion parameters, this paper proposes a new method based on the top and bottom $\mathrm{m}$-D spectra of the cone. In this method, the distribution of the m-D characteristic echo signals of the cone target is first extracted by calculating the first moment of the timefrequency. Then, the motion parameters of the target are roughly estimated by the Fourier transform of the m-D curve. Based on the rough estimation, the search method is used to accurately estimate the motion parameters of the cone target. The analytical data validates the effectiveness of the method.
Acknowledgement The guest editors are thankful to our reviewers for their effort in reviewing the manuscripts. We also thank the Editor-inChief, Dr. Imrich Chlamtac for his supportive guidance during the entire process. The special issue is sponsored by National Natural Science Foundation of China (Nos. 61401118, 61901137, 61501139 and 61671184).

Publisher's Note Springer Nature remains neutral with regard to jurisdictional claims in published maps and institutional affiliations.

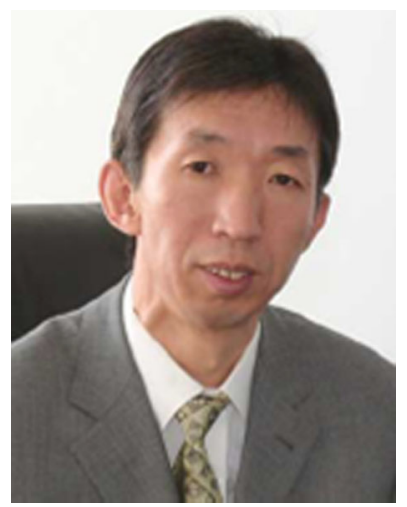

Xuemai Gu received B.E., M.E., and $\mathrm{Ph} . \mathrm{D}$. degrees in communication engineering from Harbin Institute of Technology, Harbin, China, in 1982, 1985, and 1991, respectively. He is a Professor with the Communication Research Center, Harbin Institute of Technology. He is the Dean of School of Graduate Student. He is the inventor of more than 20 China patents and has authored over 150 research papers and 5 books. His research interests include wireless networks and transmissions, private wireless communication systems, and satellite communications. 